\title{
MENINAS VELOZES: EQUIDADE DE GÊNERO EM STEM NO CONTEXTO DA BASE NACIONAL COMUM CURRICULAR
}

Ingrid de Castro Alves-190014580@aluno.unb.br

Universidade de Brasília, Instituto de Química

Kassia Glenda Sousa Silva - 180021133@aluno.unb.br

Dianne Magalhães Viana-diannemv@unb.br

Universidade de Brasília, Faculdade de Tecnologia

Carla Emanuelle Silva de Carvalho-180041495@aluno.unb.br

Katia Cristina Tarouquella Rodrigues - katia.tarouquella@unb.br

Universidade de Brasilia, Instituto de Psicologia

Simone Aparecida Lisniowski-simone.fe@unb.br

Universidade de Brasillia, Faculdade de Educação

Tânia Mara Campos de Almeida - taniamaraca@unb.br

Universidade de Brasília, Instituto de Ciências Sociais

Campus Universitário Darcy Ribeiro

70910-900 - Brasília - DF

Valerie Ganem - valerie.ganem@univ-paris13.fr

Université Sorbonne Paris-Nord, Département Gestion des Entreprises et des Administrations Avenue Jean-Baptiste Clément, 93430 Villetaneuse.

Paris - France

Resumo: Este trabalho busca compreender a importância do projeto Meninas Velozes para as estudantes do Centro de Ensino Médio 404 de Santa Maria. O estudo foi realizado a partir de entrevistas com as participantes, articulando os relatos das alunas ao debate sobre as orientações da Base Nacional Comum Curricular (BNCC) para o ensino médio buscando os pontos de intersecção entre os relatos das participantes, as ideias do projeto Meninas Velozes e os discursos de equidade e educação significativa, defendidos no texto da BNCC. O projeto Meninas Velozes foi criado em 2013 na Universidade de Brasília com a proposta de incentivar a entrada de estudantes de escolas públicas do DF no ensino nas áreas de Ciência, Tecnologia, Engenharia e Matemática (STEM). Para tanto, o projeto realiza diversas oficinas pedagógicas com foco em ciências exatas, usando metodologias de aprendizagem ativa que promovem a aprendizagem lúdica de conteúdos associados ao universo da ciência.

Palavras-chave: Meninas velozes, Metodologias ativas, STEM. 


\section{INTRODUÇÃO}

Historicamente foram construídas as bases que tornaram tanto a ciência como o gênero processos sociais e culturais reproduzidos em meio a relações de poder e de saber. O mundo das ciências exatas excluiu a presença das mulheres com um discurso biologista utilizado como justificativa para explicar a masculinização das áreas de Ciências Exatas e a feminização dos trabalhos domésticos. Assim, as dificuldades enfrentadas pelas mulheres durante toda a sua história encontram reflexo, ainda nos dias de hoje, na desigualdade de oportunidades de inserção entre gêneros na área científica, sobretudo nas Ciências Exatas.

Por exemplo, apesar de quase metade dos pesquisadores cadastrados no Conselho Nacional de Desenvolvimento Científico e Tecnológico ( $\mathrm{CNPq}$ ) serem do gênero feminino, há um desequilíbrio evidente na distribuição por áreas de conhecimento. Os dados levantados apontam que em áreas como a Linguística e a Saúde o percentual de pesquisadores $(67 \%$ e $60 \%$, respectivamente) é muito superior a áreas historicamente associadas ao universo masculino, como Engenharia e Ciências Exatas, 26\% e 33\% respectivamente (CUNHA, PERES, GIORDAN, BERTOLDO, MARQUES \& DUNCKE, 2014).

As disparidades são ainda mais expressivas conforme o nível de especialização aumenta, de modo que o número de pesquisadoras se torna consideravelmente escasso à medida que se avança na carreira científica. De acordo com dados das bolsas de produtividade do Conselho Nacional de Pesquisa do Brasil (CNPq/Brasil), no mais alto grau da carreira, pesquisador 1A, há apenas $23 \%$ de mulheres, levando-se em consideração todas as áreas de conhecimento. Já nas áreas de Ciências Exatas, a porcentagem é ainda mais baixa, chegando a 3\% (SILVA, 2020). A partir disso, Bandeira (2008, p. 208) afirma que a "pesquisa científica, ainda, está configurada primordialmente por relações sociais e por marcas culturais sexistas". Os dados apresentados nas pesquisas refletem um sistema que sistematicamente legitima a desigualdade de gênero na ciência, que estruturalmente exclui, inferioriza o papel da mulher e coloca suas características como negativas para o trabalho científico, fazendo com que as mulheres tenham que lutar, gastar seu tempo e energia para compreender os diferentes tetos de vidro e por fim conquistar espaço na ciência, insistindo neste trabalho, apoiando outras mulheres para enfrentar as dificuldades e se inserirem em áreas de predominância masculina, como as Engenharias e as Ciências Exatas.

Apoiadas nessa estrutural patriarcal, nestes espaços de pesquisa e trabalho, estão presentes práticas de assédio sexual, verbal e moral, gerando estresse e insegurança que passa a acompanhar as mulheres em toda sua trajetória acadêmica. Uma das causas para essa desigualdade entre homens e mulheres nas áreas de Ciências Exatas está associada aos estereótipos sociais, nos quais considera-se que homens e mulheres apresentam aptidões diferentes para algumas carreiras, como por exemplo a Matemática. Dessa forma, a presença das mulheres na ciência está diretamente relacionada à uma história de luta e de superação de discursos que afirmam as diferenças como determinadas por fatores biológicos ligados ao gênero, justificando assim que as mulheres sejam privadas de educação, de valorização de sua produção científica, de progressão em suas carreiras e de sua inserção no mercado de 
trabalho. Entretanto, não são fatores biológicos, mas um processo social e cultural de negação da capacidade cognitiva das meninas e que está presente inclusive no ambiente educacional. Por exemplo, na escola essa assimetria se consolida ainda mais, uma vez que garotos recebem, ainda durante a educação básica, mais estímulos para lidar com objetos e equipamentos ditos masculinos, como carros e computadores, ao passo que as garotas passam por tratamento diferente, sendo levadas a interagir com temáticas distintas, geralmente da área da saúde e da educação, campos diretamente associados ao cuidado e à proteção. Em estudos realizados em escolas, é possível perceber a separação entre os espaços de brincadeiras de meninos e meninas, a separação sexista por cores e tipos de brincadeiras legitimados para um ou outro grupo. Essas condições naturalmente influenciam na escolha da futura profissão e, consequentemente, influenciam na desarmonia entre gêneros nas áreas das Ciências Exatas, dado que há pouco incentivo para que as estudantes nelas ingressem e, quando ingressam, enfrentam estigmas, preconceitos e barreiras. Desse modo, ter um maior número de mulheres em cursos como Pedagogia e Enfermagem, e um número superior de homens em cursos como Física e Matemática mostra que ainda há uma demarcação de quais são as profissões dos homens e quais são as profissões das mulheres.

As assimetrias sociais observadas através dos espaços ocupados por homens e mulheres são oriundas não apenas de fatores econômicos. Essas desigualdades ultrapassam o âmbito doméstico e influenciam diretamente nas escolhas profissionais e na remuneração dos indivíduos. No Brasil, que possui uma história marcada pelo escravismo e o patriarcado, os marcadores sociais de gênero, classe social e raça compõem um dos determinantes da divisão social do trabalho. A divisão sexual do trabalho, compartilhada socialmente através de estereótipos de gênero, influencia diretamente nas ocupações destinadas aos homens e mulheres. Por meio desse fenômeno, certas atividades são naturalizadas enquanto femininas e masculinas, a exemplo do trabalho privado e do trabalho público e racional, respectivamente (ROCHA \& PINTO, 2018). São as mulheres que estão mais suscetíveis às piores remunerações, situação ainda mais dramática em relação às mulheres negras, condição que revela como a interseccionalidade entre esses marcadores delimitam o lugar social destinado às mulheres negras: longe do reconhecimento social e de remuneração digna (ALMEIDA, BRASIL, VIANA, LISNIOWSKI \& GANEM, 2020).

Observando a pouca representatividade feminina nas áreas $\mathrm{STEM}^{1}$, o projeto Meninas Velozes foi proposto no ano de 2013 para motivar meninas de escolas públicas para essas áreas. Inicialmente foi feito um projeto piloto de extensão em parceria com o Centro Educacional 404 de Santa Maria, escola do Distrito Federal que apresentava um alto índice de evasão escolar por parte das garotas. A escola apresentava diferentes situações que levavam à evasão por parte das meninas, como a gravidez precoce, abuso e violência, o baixo nível de renda e a necessidade de trabalhar antes de finalizar o ensino médio e poucos equipamentos e assistência do poder público que garantisse os direitos básicos e de cidadania.

\footnotetext{
${ }^{1}$ A Sigla STEM é utilizada com o intuito de unir conhecimentos de diferentes áreas para resolver problemas e desafios, significa Science, Technology, Engineering e Mathematics, que em português traduzimos para

“Ciência, Tecnologia, Engenharia e Matemática”.
} 
Desde essa primeira iniciativa, o objetivo do projeto foi promover tanto a consolidação dos conhecimentos das áreas de exatas, como também a reflexão sobre ser menina em uma região periférica e as limitações que isso acarreta. Dessa forma, são realizadas também reflexões a respeito de questões de gênero, raça e classe, buscando fortalecer a autonomia e a identidade das estudantes, assim como a ampliação de suas perspectivas de formação, constituindo um espaço de promoção da saúde mental e prevenção de conflitos psicossociais.

O projeto está apoiado em uma abordagem interdisciplinar, com atividades de ensino-aprendizagem das Ciências Naturais, Engenharia e Matemática, para estimular o interesse e capacitar as participantes para levar adiante seus estudos. Como o projeto atua na escola desde 2013, já houve o ingresso de algumas integrantes do projeto na Universidade de Brasília. Esta metodologia possibilitou oferecer uma base de apoio às secundaristas, permitindo que elas acompanhem melhor os cursos superiores e tenham suporte no projeto depois de sua inserção na universidade. Neste trabalho, buscamos compreender a perspectiva das participantes do projeto, perguntando a elas qual a contribuição do projeto. Portanto, este estudo integra uma um processo avaliativo dos impactos do projeto Meninas Velozes nas estudantes que participaram deste, considerando as barreiras e dificuldades enfrentadas na escola e na universidade. Portanto, iniciamos este trabalho discutindo os aspectos históricos do lugar da mulher na ciência, para em seguida apresentar a metodologia do trabalho e a análise dos dados da pesquisa realizada no segundo semestre de 2020 com alunas que participaram do projeto, articulando com a teoria e o debate para as orientações da Base Nacional Comum Curricular.

\section{METODOLOGIA}

Esta é uma pesquisa exploratória e qualitativa, com uso de questionários e pesquisa documental. Para a coleta de dados, utilizamos um questionário elaborado por meio da plataforma Google Forms, contendo 19 perguntas referentes aos dados pessoais das entrevistadas e questões abertas a respeito da compreensão de cada uma sobre a importância do projeto durante sua trajetória, considerando o interesse em áreas das ciências exatas e a ampliação de perspectivas futuras relacionadas ao ensino superior. O contato com as estudantes ocorreu de forma exclusivamente virtual, devido às medidas de distanciamento social causadas pela crise sanitária da Covid-19. Para tanto, contatamos a última turma de alunas (formandas de 2019) do Centro de Ensino médio 404 de Santa Maria que participaram do projeto, e, por meio de um grupo de WhatsApp, convidamos as alunas para contribuírem com a pesquisa. A solicitação encaminhada a 12 estudantes e destas, 5 meninas responderam.

Alguns trechos dos depoimentos coletados por meio do questionário foram transcritos para a análise e discussão do presente trabalho, preservando o anonimato das participantes e, portanto, seus nomes foram omitidos.

Para o desenvolvimento da pesquisa documental, analisamos a versão da Base Nacional Comum Curricular disponível no site do Ministério da Educação $^{2}$ até a presente data (julho/2020), especificamente as seções 1, uma introdução, e 5, relativa ao ensino médio.

\footnotetext{
${ }^{2}$ Disponível no site: http://basenacionalcomum.mec.gov.br/
} 
Ademais, realizamos um levantamento bibliográfico das publicações feitas sobre o projeto Meninas Velozes, assim como de temas relacionados à educação e à equidade de gênero.

As seções da Base estão inseridas em um documento de seiscentas páginas, divididas em cinco capítulos. O primeiro consiste numa introdução, o segundo apresenta a estrutura da elaboração da BNCC, o terceiro mostra as contribuições para o ensino infantil, o quarto apresenta as colocações realizadas a respeito do ensino fundamental e cabe ao quinto a discussão sobre o ensino médio no contexto da educação brasileira.

Buscamos, como objetivo de pesquisa, analisar essas relações entre os discursos de equidade e educação significativa, defendidos no texto da BNCC, as ideias do projeto Meninas Velozes e os relatos das alunas integrantes do projeto que participaram da pesquisa neste momento. A seguir contextualizamos o projeto apresentando seu histórico, metodologia e objetivos e apresentamos a análise dos dados.

\section{CONTEXTUALIZAÇÃO DO PROJETO MENINAS VELOZES E ANÁLISE DOS DADOS}

Para a realização deste projeto participam algumas professoras da Faculdade de Tecnologia da Universidade de Brasília, Departamento de Engenharia Mecânica, professoras de Engenharia do campus UnB/ Gama e professoras das áreas de Educação, Sociologia e Psicologia, que orientam as alunas de graduação dos respectivos cursos como Engenharias, Química, Sociologia e Psicologia. Como é um projeto de extensão junto à escola, há a colaboração da direção da instituição de ensino e de um professor responsável pelas estudantes de Ensino Médio.

Dentro do projeto existem diferentes mecanismos de atuação, ou seja, diferentes atividades que são desenvolvidas com o comum objetivo: incentivar as alunas de ensino médio para as diferentes possibilidades de atuação dentro das ciências exatas. Para isso, as alunas de graduação são responsáveis por desenvolver o que chamamos de oficinas. Tais oficinas são encontros com as alunas de ensino médio, nos quais são abordados os conteúdos estudados em matérias como matemática, física e química de uma forma aplicada, ativa e descontraída. Além disso, são feitas rodas de conversas referentes às temáticas de gênero e educação, assim como a realização de visitas educativas em diversos espaços, como os laboratórios de engenharia da UnB.

A realização das oficinas pedagógicas é embasada em estratégias e metodologias de aprendizagem ativa, como práticas hands on e Aprendizagem Baseada em Problemas, envolvendo situações do cotidiano das alunas de ensino médio, e também apresentando novas perspectivas relacionadas às áreas de STEM.

Com relação às oficinas, quando perguntado às alunas que participaram do projeto no ano de 2019, através do questionário aplicado sobre a relevância dessas atividades, elas enfatizam o aspecto lúdico e de aprendizagem ativa. Para ilustrar apresentamos dois depoimentos: "As oficinas eram completamente interessantes pois me divertia e também se tratava de uma forma de aprendizado", "Ver em prática conceitos teóricos aprendidos na escola fazem total diferença no entendimento do assunto". 
Segundo Moraes \& Manzini (2006), a Aprendizagem Baseada no Problema (ABP), diferentemente das concepções hegemônicas de ensino, cujo foco principal é o pressuposto de transmissão do conhecimento, baseia-se em um método de ensino-aprendizagem construtivista, no qual o aluno deixa de ser um agente passivo e torna-se protagonista desse processo. Do mesmo modo, o professor deixa de ser unicamente um agente transmissor de conhecimento e passa a valorizar os conhecimentos e habilidades prévias de cada estudante. Tendo como referência esse mecanismo de atuação, o projeto Meninas Velozes se tornou uma importante ferramenta na quebra de estereótipos em relação à atuação dentro das Engenharias e Ciências Exatas por ter esse propósito de aproximar e familiarizar as participantes às áreas citadas.

Quando perguntadas se a visão em relação às áreas de Ciências Exatas mudou após participar do projeto, 4 dentre as 5 estudantes responderam que sim, a visão mudou.

Verificamos que o projeto se constitui um importante fator motivacional para essas alunas, uma vez que permite o contato com mulheres atuantes nas áreas de Ciências Exatas, evidenciando que é possível ocupar diferentes espaços (ALMEIDA, BRASIL, VIANA, LISNIOWSKI \& GANEM, 2020). Revelando ser um agente de mudança de perspectiva de futuro após o final do ensino médio, como mostra a fala de algumas alunas ex-participantes do projeto: "O projeto ampliou os meus horizontes", "O projeto foi importante para mim principalmente ao quebrar o estereótipo que tinha das ciências exatas, como uma coisa monótona."

\section{AS PERSPECTIVAS DO PROJETO MENINAS VELOZES DIANTE DA BASE NACIONAL COMUM CURRICULAR}

A Base Nacional Comum Curricular, BNCC, consiste em um documento normativo cuja finalidade é estipular as agregações que todos os alunos devem desenvolver ao longo da Educação Básica. Sendo parte integrante do Plano Real da Educação, previsto na Constituição Federal de 1988, orienta-se por princípios que almejam a construção de uma sociedade igualitária, valorizando, para tanto, uma formação humana. Este documento funciona, na prática, como instrumento de combate à normalização da desigualdade educacional entre raça, sexo e condição econômica, perpetuada historicamente no Brasil.

A BNCC foi elaborada por profissionais de diversas áreas, redigida pela primeira vez em 2014, passando por diversas modificações, sendo aprovada apenas em 2017, após ter sido aberta para consulta e sugestões do público, em um processo que mobilizou cerca de quarenta e cinco mil escolas (FERREIRA, 2020). Visto que procura moldar-se às sugestões dos cidadãos, apresenta caráter coletivo, pois permite, ainda, que cada instituição adapte suas particularidades à proposição geral que o norteia: a de ser balizador da qualidade educacional, bem como atender às necessidades e realidades do aluno contemporâneo, não esquecendo, contudo, de prepará-lo para o futuro e reconhecendo, por fim, que a educação busca a reflexão crítica e promover processos educacionais que contribuam para a transformação da sociedade. Portanto, mostra-se uma ferramenta essencial quanto à superação da desigualdade entre gêneros na esfera científica, uma vez que se preocupa com a ampliação da educação brasileira 
e busca atenuar os resultados de uma sociedade misógina que perpetua a exclusão feminina desde o Ensino Básico às atividades culturalmente destinadas aos meninos e aos homens.

Em concordância com a proposta do registro, dez competências gerais, definidas no referido documento como habilidades, valores e conhecimentos para solucionar as imposições cotidianas, devem ser asseguradas. Constata-se que na BNCC (BRASIL, 2018, p. 09) a segunda competência é a pesquisa, tem como objetivo exercitar a curiosidade intelectual e recorrer à abordagem própria das ciências, incluindo a investigação, a reflexão e análise crítica para elaboração de hipóteses, formulação e resolução de problemas nas diversas áreas do conhecimento.

Por sua vez, a sexta competência (BRASIL, 2018, p. 09) defende a valorização dos diversos conhecimentos e saberes culturais, e da apropriação, por parte do aluno, de experiências que lhe possibilitarão fazer escolhas que estejam equiparadas ao seu plano de vida. Nessa conjuntura, o Meninas Velozes apresenta-se, por conseguinte, consoante à Base Nacional Comum Curricular, uma vez que, por meio de rodas de conversa e debates sobre questões relacionadas à identidade, promove a reflexão acerca da perspectiva indivíduo-sociedade, incentivando as participantes a atingirem objetivos pessoais, assim como torna possível a troca de experiência entre alunas do ensino médio, graduandas e professoras.

As estratégias e metodologias de aprendizagem ativa do projeto centralizam-se na ideia de dar sentido e contexto ao que se aprende, estimulando, desse modo, o protagonismo das estudantes, tal como esperado pelas diretrizes pedagógicas do referido documento.

Tendo em vista o caráter obrigatório da Base Nacional Comum Curricular, publicada em 2018, e sua implementação, que está prevista para o Ensino Médio, o foco do projeto Meninas Velozes, a partir de 2021, é direcionar-se à fortificação das sessões de debate, a fim de valorizar a diversidade e acolher as diferenças, além de permitir um olhar crítico para o mundo. Com vistas a ser sensível às demandas das jovens, será adotado o uso de questionários esporádicos, de modo a aumentar a interação entre as participantes e uma avaliação contínua das atividades, decorrendo em maior compreensão sobre suas experiências e expectativas para com o projeto. Esses indicadores também serão utilizados como um indutor das mudanças necessárias, na medida em que conferem protagonismo às participantes ao introduzi-las no processo criativo de novas oficinas e atividades, conforme estimula a BNCC quando sinaliza a necessidade de romper com a imagem do professor enquanto único e principal transmissor de conhecimento e prioriza sua função de mediador de uma educação centrada no aluno, aliando a esse processo o uso da tecnologia.

Portanto, a BNCC pode revelar-se um plano importante e necessário no combate às diferenças entre gêneros na educação, assim esperamos que possa contribuir para que novos projetos desta natureza sejam implementados e articulados à escola. O projeto Meninas Velozes é, nessa concepção, relevante na perspectiva das alunas e na articulação com um documento norteador das ações na escola. Uma proposta que está em constante reformulação para melhor atender aos objetivos de equidade de gênero na escola e na universidade. A proposta do projeto no escopo da BNCC é apontar para a escola que deseja consolidar e ampliar oportunidades de aprendizagem e de inclusão, já que tem como objetivo promover a equidade e, para tanto, promover o ensino interativo, tornando a o processo educacional mais significativo. 


\section{CONSIDERAÇÕES FINAIS}

Este foi um primeiro passo para um estudo mais aprofundado sobre a perspectiva das alunas participantes acerca do projeto e a busca de implementar a concepção e propostas da BNCC na escola. Acreditamos que é possível integrar nas atividades os objetivos da base curricular considerando principalmente o desenvolvimento de competências e habilidades nas Ciências Exatas e a busca de equidade de gênero. Tendo em vista os pontos abordados, observamos que o projeto Meninas Velozes, por meio de sua atuação em uma escola periférica do Distrito Federal, representa uma importante iniciativa na perspectiva das participantes, assim como integra com relação à BNCC a desconstrução de estereótipos de gênero relacionados ao ambiente acadêmico e aos espaços destinados aos homens e às mulheres.

Acreditamos que a Universidade, por meio de projetos de extensão, tem um importante papel na promoção de ações voltadas à reparação dessas desigualdades, desenvolvendo projetos como o Meninas Velozes, uma vez que as oficinas pedagógicas, realizadas regularmente sobre temas relacionados aos conteúdos das Ciências Exatas, permitem que as alunas entrem em contato, de forma ativa e lúdica, com áreas muitas vezes consideradas inacessíveis para as mulheres. Além disso, o contato com profissionais e professoras atuantes na área e com as monitoras, cuja faixa etária é próxima a das alunas, permite a estas vislumbrar a área de exatas como um universo possível e não unicamente restrito aos homens.

Alinhado a essa necessidade, observamos que é importante revisar periodicamente o projeto Meninas Velozes, com o propósito de avaliar seu desempenho e identificar outras formas de planejamento e execução das oficinas, assim como aprimorar a concepção do projeto e integrá-lo à outras propostas similares na escola.

$\mathrm{O}$ projeto Meninas Velozes se constitui, portanto, enquanto um importante fator motivacional para que as alunas ampliem suas perspectivas em relação ao ensino superior, bem como em relação às Ciências Exatas, como elucidado em trechos das respostas coletadas por meio do questionário aplicado, em que as participantes destacam o incentivo ao protagonismo feminino, o acolhimento da universidade pública e a identificação com as Engenharias.

Esperamos, com a divulgação deste trabalho, instigar outras iniciativas de extensão e pesquisa sobre desigualdade de gênero, raça e classe social nas escolas. Este trabalho pretende se ampliar e abordar outros aspectos da desigualdade de gênero nas Engenharias, que aqui não foram mencionados, mas que são pertinentes para alcançar uma compreensão global sobre tal condição e, por conseguinte, são salutares para desenvolver outras estratégias e instrumentos para lidar com esse problema também na universidade.

\section{Agradecimentos}

Agradecemos o empenho e dedicação de todas as professoras que estão na linha de frente do projeto e ao apoio por nós recebido. À Fundação de Apoio à Pesquisa do Distrito Federal, FAP - DF, por nos proporcionar recursos para que a nossa atuação seja possível. 
Agradecemos também o cuidado de todas as monitoras e estudantes de graduação que atuam no projeto, que tornam o ambiente mais colaborativo e possibilita trocas de experiências. Finalmente, ao Centro de Ensino Médio 404 e aos professores da instituição, por colaborarem e tornarem possível a execução deste projeto.

\section{REFERÊNCIAS}

ALMEIDA, Tania Mara Campos de, BRASIL, Katia Tarouquella, VIANA, Dianne Magalhães, LISNIOWSKI, Simone, \& GANEM, Valérie. A passos largos: meninas da periferia rumo à universidade e seus dilemas psicossociais. Sociedade e Estado, Brasília, v. 35, n.1, p.101-134, jan. 2020. Disponível em: http://www.scielo.br/scielo.php?script=sci_arttext\&pid=S0102-69922020000100101\&lng=pt \&nrm=iso. Acesso em: 17 jun. 2020.

BANDEIRA, Lourdes. A contribuição da crítica feminista à ciência. Estudos Feministas, Florianópolis, 16(1): 288, janeiro-abril/2008.

BARROS, S. C. da V. e MOURÃO, L. Existem Diferenças de Gênero Entre os Bolsistas Produtividade do Conselho Nacional de Desenvolvimento Científico e Tecnológico (CNPq)?

Disponível

em:

https://humanas.blog.scielo.org/blog/2020/05/08/existem-diferencas-de-genero-entre-os-bolsi stas-produtividade-do-conselho-nacional-de-desenvolvimento-cientifico-e-tecnologico-cnpq/. Acesso em: 21 jun. 2020.

BRASIL. Base Nacional Comum Curricular (BNCC). Educação é a Base. Brasília, MEC/CONSED/UNDIME, 2018. Disponível em:

http://basenacionalcomum.mec.gov.br/images/BNCC_publicacao.pdf. Acesso em: 02 jul. 2020.

CUNHA, M. B; PERES, O.M; GIORDAN, M; BERTOLDO, R.R; MARQUES, G.Q; DUNCKE, A. C. As Mulheres na Ciência: O Interesse das Estudantes Brasileiras pela Carreira Científica. In: Mujeres en la Química, 2014. Universidad Nacional Autónoma de México, 2014. p. 1-11.

FERREIRA, Felipe. Entenda como funciona a Base Nacional Comum Curricular (BNCC). IN: Proesc Blog: Gestão Pedagógica. Publicado em setembro de 2020. Disponível em: http://www.proesc.com/blog/entenda-a-base-nacional-comum-curricular-bncc/. Acesso em: set. 2020 .

MORAES, Magali Aparecida Alves de; MANZINI, Eduardo José. Concepções sobre a aprendizagem baseada em problemas: um estudo de caso na Famema. Revista Brasileira de Educação Médica, Rio de Janeiro, v. 30, n. 3, p. 125-135, Dec. 2006. Disponível em: 
http://www.scielo.br/scielo.php?script=sci_arttext\&pid=S0100-55022006000300003\&lng=en $\&$ nrm=iso. Acesso em 17 jul. 2020.

MORAN, J. M. Mudando a educação com metodologias ativas. In Convergências Midiáticas, Educação e Cidadania: aproximações jovens. Coleção Mídias Contemporâneas. 2015 Disponível em:

http://www2.eca.usp.br/moran/wpcontent/uploads/2013/12/mudando_moran.pdf. Acesso em: 07 ago. 2020.

ROCHA, Euda Kaliani Gomes Teixeira; PINTO, Francinaldo do Monte. O desafio conceitual do trabalho doméstico à psicologia do trabalho. Revista de Psicologia, v. 30, n. 2, 2018, p. 145-153.

SILVA, Gabriella Galdino da et al . Tem Menina no Circuito: dados e resultados após cinco anos de funcionamento. Rev. Bras. Ensino Fís., São Paulo， v. 42， e20200328， 2020 . Disponível em:

$<$ http://www.scielo.br/scielo.php?script=sci_arttext\&pid=S1806-11172020000100485\&lng=e $n \& n r m=$ iso $>$. access on 01 Oct. 2020. Epub Sep 23,

2020. https://doi.org/10.1590/1806-9126-rbef-2020-0328.

\title{
FAST GIRLS: GENDER EQUITY IN STEM IN THE CONTEXT OF THE COMMON NATIONAL CURRICULUM BASE
}

\begin{abstract}
The sexual division of labor, shared socially by means of gender roles, directly impacts the occupation of women and men in various spaces. While women are naturalized as activities related to care and home, the men are the most socially recognized activities performed in the public sphere. Seeking to intervene in this reality, the project called "Meninas Velozes"' (Fast Girls) was born in 2013 in a peripheral school in the Federal District (404 High School Center of Santa Maria) in partnership with the University of Brasilia with the proposal to encourage the entry of students in higher education and careers typically aimed for men, the areas of Science, Technology, Engineering and Mathematics (Stem). To this end, the project conducts several pedagogical workshops focused on the science areas, a methodology that allows students access to contents associated with the universe of science. In each workshop, high school students live in practice the use of the contents of the exact science subjects studied in the classroom in an active and playful way. In addition, it aims to promote the mental health of students from this space, through debates and conversation wheels focused on gender and education issues. From this perspective, the article aims is to analyze the importance of project "Meninas Velozes "for the students of the 404 High School Center of Santa Maria.
\end{abstract}

Keywords: Fast Girls. Active Methodology. STEM. 